\title{
EXPERIMENTAL DETERMINATION OF THE LOW-LYING EXCITED A STATES OF trans-STILBENE
}

\author{
GEORG HOHLNEICHER and BERNHARD DICK ${ }^{\dagger}$ \\ Lehrstuhl für Theoretische Chemie der Universität zu Köln, Greinstrasse 4, 5000 Cologne \\ 41 (F.R.G.)
}

(Received December 8, 1983)

\section{Summary}

The low-lying A states of trans-stilbene are investigated by means of two-photon excitation spectroscopy. The experimental findings are compared with theoretical results. From the combined information of oneand two-photon spectroscopy we can assign at least seven excited singlet states in the energy range below $50000 \mathrm{~cm}^{-1}$. The newly obtained information is discussed with regard to the mechanism proposed for the photoisomerization of stilbene.

\section{Introduction}

The photoisomerization of stilbene certainly belongs to the most extensively studied photochemical reactions. The detailed understanding of this reaction has always been regarded as a major goal by photochemists and photophysicists. The model now generally accepted to describe the basic pattern of the photoisomerization of stilbene along the singlet pathway has been proposed by Orlandi and Siebrand [1]. In this model the first excited singlet state $1 \mathrm{~B}$, which gives rise to the first strong absorption band at about $30000 \mathrm{~cm}^{-1}$ in the UV spectrum of trans-stilbene, is assumed to increase in energy when the angle $\phi$ of rotation around the central double bond increases (Fig. 1(a)). However, since the ground state of the trans form correlates with a doubly excited configuration of the cis form and vice versa, an avoided crossing must take place near $\phi=90^{\circ}$. This avoided crossing causes the well-known barrier in the ground states $S_{0}$ (about $49 \mathrm{kcal} \mathrm{mol}^{-1}$ for trans $\rightarrow$ cis isomerization [2]) and thus inevitably leads to a low-lying excited A state in the vicinity of $\phi=90^{\circ}$. This state has been called the "phantom state" ${ }^{1} \mathrm{p}$ "* (where $\mathrm{p}$ stands for perpendicular) by Saltiel et al. [3]. In the Orlandi-Siebrand (OS) model the corresponding state is termed S2 since it is assumed to lie above $1 \mathrm{~B}$ at $\phi=0^{\circ}$ and $\phi=180^{\circ}$. At $\phi=90^{\circ}$,

TPresent address: Abteilung Laserphysik, Max-Planck-Institut für Biophysikalische Chemie, Am Fassberg, D-3400 Göttingen, F.R.G. 


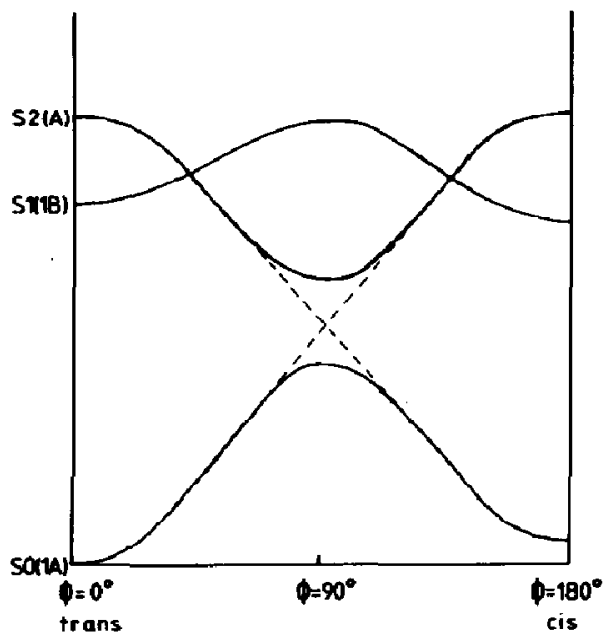

(a)

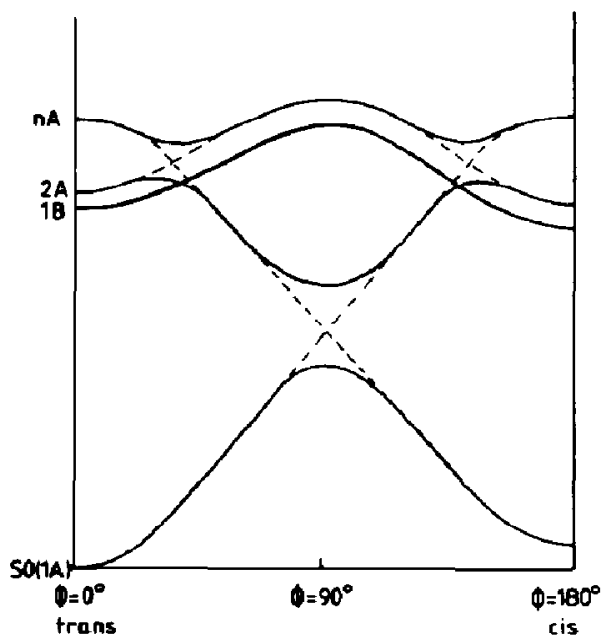

(b)

Fig. 1. Possible models for the photoisomerization of stilbene (see text).

however, $\mathrm{S2}$ is expected to be the lowest excited singlet state as shown in Fig. 1(a). Photoisomerization is then believed to proceed via a non-adiabatic transition between the two potential surfaces followed by rapid internal conversion to $S_{0}$ in the vicinity of $\phi=90^{\circ}$.

The OS model explains quite well a variety of experimental observations [1,2-12] especially the appearance of a barrier which has to be overcome when isomerization starts from the lowest excited singlet state of the trans form (experimental value of about $3.5 \mathrm{kcal} \mathrm{mol}^{-1}$ in the gas phase [11] as well as in solution [10]). A major disadvantage of the OS model, however, is that it makes use of states we do not know experimentally. In particular, we do not know to which excited A state of the trans or cis form ${ }_{1}^{1} p^{* *}$ really corresponds. The lowest excited A state (2A) of the trans and cis form most probably does not correspond to ${ }^{1} \mathrm{p}^{* *}$. In going from $\phi=0^{\circ}$ and $\phi=180^{\circ}$ towards $\phi=90^{\circ}$ the $2 \mathrm{~A}$ state is expected to increase in energy in a similar way as does 1B [13-18] (Fig. 1(b)). Thus, we have to anticipate an avoided crossing between this $A$ state and the $\mathrm{S} 2$ state of the OS model. The latter probably corresponds to one of the higher excited A states in the trans and in the cis form. The avoided crossing may well cause a barrier in the lowest excited A state 2A itself, as shown in Fig. 1(b). The question then arises whether the experimentally observed barrier $[6,10,11]$ is really due to the non-adiabatic crossing from $1 \mathrm{~B}$ to $2 \mathrm{~A}$ or probably due to the barrier in $2 \mathrm{~A}$.

To gain a better insight into this puzzling situation, we have investigated the two-photon excitation (TPE) spectrum of trans-stilbene in the energy range $29000-49000 \mathrm{~cm}^{-1}$. This spectrum should yield information specifically on states of symmetry $A$. We have also studied the polarization of the one-photon spectrum of stilbene and a closely related compound to clarify some inconsistencies in connection with the assignment of the second 
UV band. The spectra obtained are compared with earlier measurements of two-photon absorption $[19,20]$ and with the results of calculations. Finally, the implications on the OS model are discussed.

\section{Experimental details}

The UV spectra were measured on a UV spectrometer Beckmann Acta VI. For the spectra measured at $77 \mathrm{~K}$ a commercially available low temperature cell was used.

The polarization of the one-photon excitations was studied with the method of luminescence polarization [21]. The degree $P$ of polarization obtained with this method is related to the angle $\alpha$ between the transition moments of emission and absorption by

$P=\frac{3 \cos ^{2} \alpha-1}{\cos ^{2} \alpha+3}$

Under ideal conditions (no overlapping bands, no vibronic perturbation) $P$ is 0.5 for parallel and $-1 / 3$ for perpendicular oscillators. $P$ was measured as a function of the excitation energy (the absorption polarization spectrum) with a home-made instrument [22] following the method introduced by Dehler and Dörr [23].

TPE spectra for circularly polarized laser light and linearly polarized laser light with two-photon cross sections $\delta_{\text {TC }}$ and $\delta_{\uparrow \uparrow}$ respectively were obtained with an experimental set-up which is described in detail in ref. 24 . Data points were taken in steps of $5 \AA$ in the range $4000-6900 \AA$. The sample was trans-stilbene in ethanol solution (about $10^{-3} \mathrm{M}$ ) at room temperature. The two-photon polarization parameter

$\Omega=\frac{\delta c c}{\delta_{\uparrow \uparrow}}$

which can only have values in the range between 0 and $3 / 2$ was used as a symmetry indicator [25].

The symmetries for different geometries of stilbene are given in Table 1. When only next-neighbour interactions and the orientation of the $\pi$ lobes are considered, higher symmetries $\left(D_{2 h}, D_{2}\right.$ and $\left.D_{2 d}\right)$ are derived, which we refer to as "topological symmetries". For a molecule such as stilbene, where the deviation from the topological symmetry is not very large, the electronic states clearly resemble those in the corresponding higher symmetry.

The components of the two-photon transition tensor transform as the products of the coordinates. For all final states with B symmetry the diagonal elements of the two-photon transition tensor vanish. All allowed twophoton transitions to final B states should therefore have an $\Omega$ value of $3 / 2$ [25]. For allowed two-photon transitions to final A states usually a much lower $\Omega$ value is found, often close to $2 / 3[24,26]$. This value indicates that one diagonal element is dominant $[25,27]$. For molecules belonging to 
TABLE 1

Symmetries for the different possible geometries of stilbene ${ }^{a}$

Molecular symmetry

$C_{2 h}\left(\phi=0^{\circ} ; \theta=0^{\circ}, 180^{\circ}\right)$

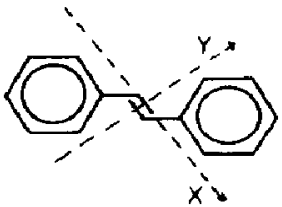

$\mathbf{A}_{\mathbf{g}}$

$x^{2}, y^{2}, z^{2}, x y \quad z$

$\mathbf{A}_{\mathbf{u}} \quad \mathbf{B}_{\mathbf{g}}$

$\mathbf{B}_{\mathbf{u}}$

$z \quad x z, y z \quad x, y$

$C_{2}\left(\phi\right.$ and/or $\left.\theta \neq 0^{\circ}, 180^{\circ}\right)$

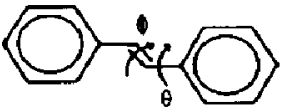

$C_{2 v}\left(\phi=180^{\circ} ; \theta=0^{\circ}, 180^{\circ}\right)$

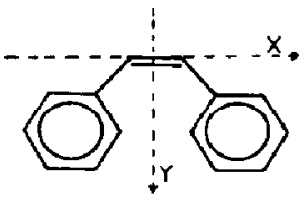

$\mathbf{A}_{1}$

$z, x^{2}, y^{2}, z^{2}$

$\mathbf{A}_{2} \quad \mathbf{B}_{1}$

$\mathbf{B}_{2}$

$z, x^{2}, y^{2}, z^{2}, x y$

$x, y, x z, y z$

B

A

$\boldsymbol{x y} \quad \boldsymbol{x}, \boldsymbol{x} z$

$\boldsymbol{y}, \boldsymbol{y z}$

Topological symmetry

$D_{2 h}\left(\phi=0^{\circ} ; \theta=0^{\circ}, \mathbf{1 8 0}^{\circ}\right)$

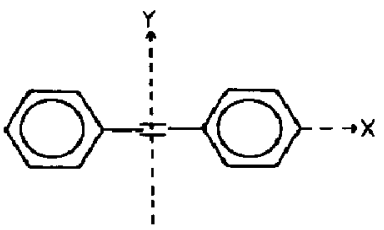

$\begin{array}{llllllll}\mathbf{A}_{\mathrm{g}} & \mathbf{B}_{1 \mathrm{~g}} & \mathbf{B}_{2 \mathrm{~g}} & \mathbf{B}_{3 \mathrm{~g}} & \mathbf{A}_{\mathrm{u}} & \mathbf{B}_{1 \mathrm{u}} & \mathbf{B}_{2 \mathrm{u}} & \mathbf{B}_{3 \mathrm{u}} \\ x^{2}, y^{2}, z^{2} & x y & x z & y z & - & z & y & x\end{array}$

$D_{2}\left(\phi \neq 0^{\circ}, 90^{\circ}, 180^{\circ}\right.$

and/or $\theta \neq 0^{\circ}, 180^{\circ}$ )

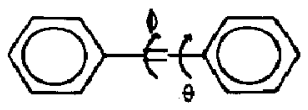

A

$B_{1} \quad B_{2}$

$\mathbf{B}_{3}$

$x^{2}, y^{2}, z^{2}$

$z, x y$

$y, x z$

$x, y z$

$D_{2 d}\left(\phi=90^{\circ} ; \theta=0^{\circ}, 180^{\circ}\right)$
$\mathbf{A}_{1}$
$\mathbf{A}_{2} \quad \mathbf{B}_{1}$
$\mathbf{B}_{2} \quad \mathbf{E}$
$x^{2}+y^{2}, z^{2} \quad-\quad x^{2}-y^{2} \quad z, x y \quad x, y, x z, y z$

Only symmetrical twists of the phenyl rings are considered. The $x$ axis is taken in the direction of the central $\mathrm{C}=\mathrm{C}$ bond. Thus the $x$ axis is the same for all possible geometries. It should be noted that this leads to an unconventional labelling of the axis in cis-stilbene. 
point groups $C_{2 h}$ and $C_{2}$, however, the tensor element which transforms as $x y$ belongs to $A_{g}$ and $A$ respectively. If this element is dominant for a given transition, the $\Omega$ value will be close to $3 / 2$ in spite of the fact that the final state is an $A$ state. This may happen if the final state evolves mainly from a state which is $B_{1 g}, B_{1}$ or $B_{2}$ in the corresponding topological symmetry.

In solution the ground state of trans-stilbene is certainly not planar. The angle $\theta$ by which the phenyl rings are twisted with respect to the plane of the central double bond is assumed to be somewhat smaller $[13,28]$ than in the gas phase $\left(\theta=33^{\circ}[29]\right)$. As a result of this non-planarity the mutual exclusive selection rules for one- and two-photon allowed transitions which hold for planar trans-stilbene (point group, $C_{2 h}$ ) are no longer valid and two-photon transitions to those final $B$ states which are responsible for the prominent bands in the one-photon spectrum $[13,28]$ may gain some intensity.

\section{Calculations}

To support our interpretation and assignment of excited singlet states, we have performed complete neglect of differential overlap/S (CNDO/S) calculations, both in the original [30] and in an extended [27,31] version (Table 2). The standard CNDO/S procedure was employed with 60 singly excited configurations using the Mataga-Nishimoto approximation [32] for electron repulsion integrals (SCI/M 60). The extended scheme includes 200 energy-selected singly and doubly excited configurations (SDCI) and makes use of the Pariser-Parr formula [33]. All other parameters in both procedures are taken from the final parameter set of the CNDO/S method [34]. Two-photon cross sections are calculated using a value of $5 \times 10^{-15} \mathrm{~s}$ for the line shape function [27].

The results of two SDCI calculations are presented for planar transstilbene: one in which only $\pi \pi^{*}$ excitations are included (SDCI/P 200 $\pi^{*}$ ) and one which also takes into account other excitations (SDCI/P 200). The SDCI/P 200 $\pi \pi^{*}$ scheme was found to give very valuable two-photon cross sections in recent applications [26]. The input geometry was obtained from $X$-ray data [35]. The differences between the two calculations are only minor. In the SDCI/P $200 \pi \pi^{*}$ calculation the excitation energies are shifted more or less parallel by approximately $2500 \mathrm{~cm}^{-1}$ towards higher energy as a result of the stronger stabilization of the ground state (a discussion of this problem is given in ref. 16). As in the other examples studied recently [26] the calculated absolute $\delta$ values become smaller with extension of the configuration interaction, but the relative values do not change very much.

To study the influence of non-planarity, results of an SDCI/P 200 calculation with phenyl rings twisted symmetrically by $\theta=20^{\circ}$ are given in Table 3 . The bond lengths and bond angles are the same as in the planar form. For the lowest eight excited states the influence of non-planarity is 


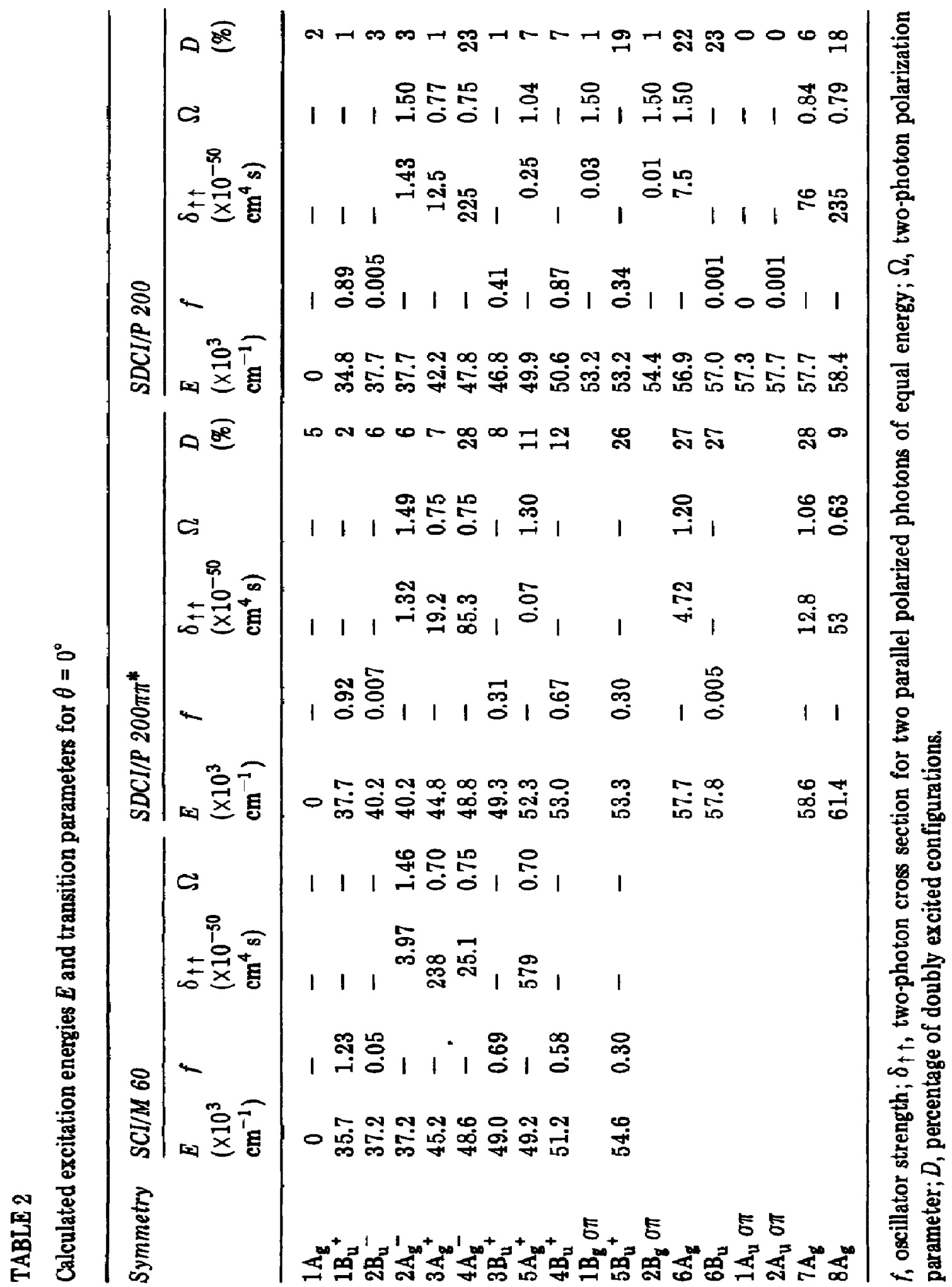


TABLE 3

Calculated excitation energies $E$ and transition parameters for $\theta=20^{\circ}$

\begin{tabular}{|c|c|c|c|c|c|}
\hline \multirow{2}{*}{ Symmetry } & \multicolumn{5}{|l|}{$S D C I / P 200$} \\
\hline & $\begin{array}{l}E \\
\left(\times 10^{3} \mathrm{~cm}^{-1}\right)\end{array}$ & $f$ & $\begin{array}{l}\delta_{\uparrow \uparrow} \\
\left(\times 10^{-50} \mathrm{~cm}^{4} \mathrm{~s}\right)\end{array}$ & $\Omega$ & $D(\%)$ \\
\hline $1 \mathrm{~A}$ & 0 & - & - & - & $\mathbf{1}$ \\
\hline $1 \mathrm{~B}^{+}$ & 36.1 & 0.90 & 0.0001 & 1.50 & 1 \\
\hline $2 \bar{B}^{-}$ & 38.1 & 0.007 & 0.21 & 1.50 & 2 \\
\hline $2 A^{-}$ & 38.1 & 0.0007 & 1.0 & 1.50 & 2 \\
\hline $3 \mathbf{A}^{+}$ & 42.4 & 0.0002 & 8.1 & 0.77 & $\mathbf{1}$ \\
\hline $4 \mathrm{~A}^{-}$ & 48.1 & 0.0001 & 197 & 0.75 & 21 \\
\hline $3 \mathbf{B}^{+}$ & 46.9 & 0.49 & 0.03 & 1.50 & 1 \\
\hline $5 \mathrm{~A}^{+}$ & 50.9 & 0.11 & 0.07 & 0.97 & 7 \\
\hline $4 B^{+}$ & 51.2 & 0.74 & 0.01 & 1.50 & 7 \\
\hline $5 B$ & 52.7 & 0.12 & 0.24 & 1.50 & 12 \\
\hline $6 \bar{B}$ & 54.0 & 0.09 & 0.08 & 1.50 & 1 \\
\hline $7 \mathrm{~B}$ & 56.2 & 0.22 & 0.23 & 1.50 & 5 \\
\hline $6 \mathrm{~A}$ & 57.2 & 0.001 & 7.4 & 1.36 & 15 \\
\hline $8 B$ & 57.3 & 0.03 & 1.4 & 1.50 & 17 \\
\hline $7 \mathrm{~A}$ & 57.7 & 0.0002 & 49 & 0.88 & 5 \\
\hline $8 \mathrm{~A}$ & 58.1 & 0 & 14 & 0.85 & 2 \\
\hline $9 A$ & 58.4 & 0 & 6.3 & 0.84 & 2 \\
\hline $10 \mathrm{~A}$ & 58.6 & 0.0002 & 129 & 0.83 & 15 \\
\hline
\end{tabular}

small in contrast with the results obtained by Olbrich [18]. Only in the region where the first $\sigma \pi^{*}$ excitations appear in the planar form $\left(1 \mathrm{~B}_{\mathrm{g}}\right.$ and $2 \mathrm{~B}_{\mathrm{s}}$ ) do deviations between the two calculations become obvious. As expected, two-photon transitions to states evolving from $B_{u}$ states of the planar trans form are no longer forbidden but their intensity is still very low.

As far as possible a pseudoparity classification "plus" or "minus" is assigned to the low-lying excited states [36] in spite of the fact that the pairing theorem does not hold in the CNDO/S method [37]. No multiplicity index is supplied with state symbols since we deal only with singlet states in this study (for information on photoisomerization along the triplet pathway see refs. 2 and 3 ).

\section{Results}

The TPE spectrum of trans-stilbene is shown in Fig. 2. In Fig. 3 the TPE spectrum is compared with the one-photon spectrum on a logarithmic scale. Included in Fig. 3 are the UV spectra of trans-stilbene and transindanylidenindane measured in 3-methylpentane at $77 \mathrm{~K}$. In trans-indanylidenindane the angle $\theta$ is expected to be close to zero because of the presence of the five-membered rings. The UV spectra of trans-stilbene and transindanylidenindane are very similar (only the vibrational structure is more 
(a)

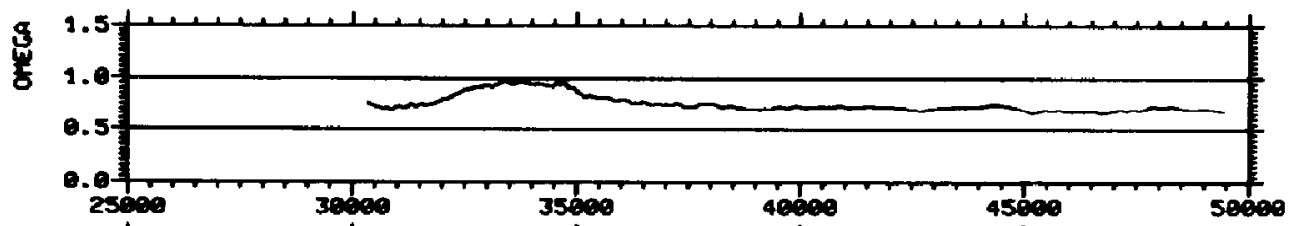

(b)

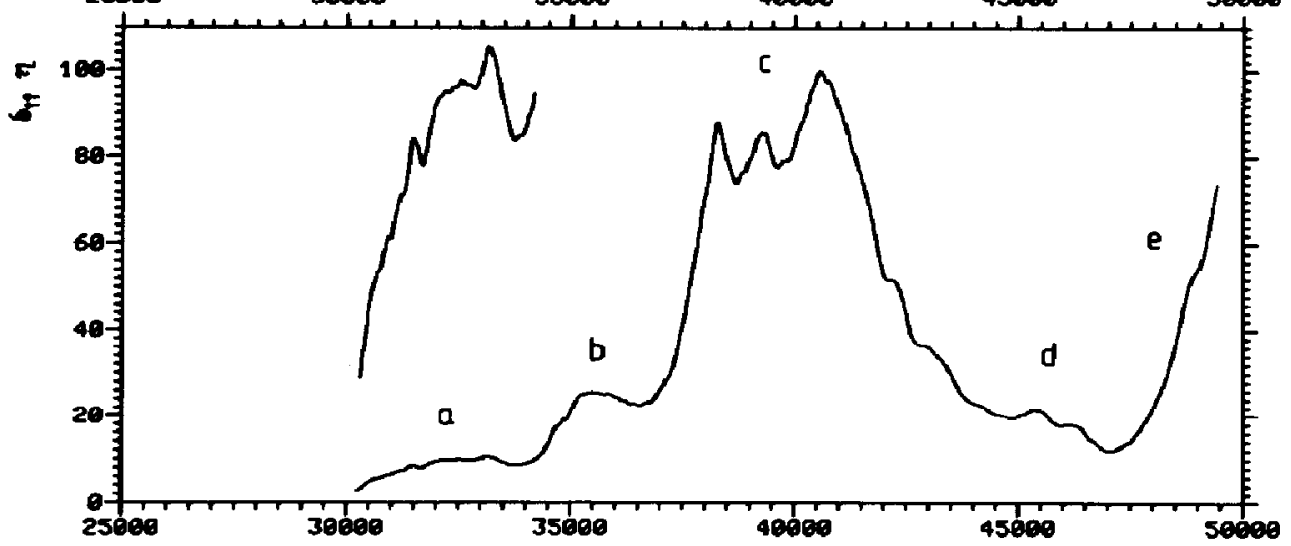

HAUEMUMBER

Fig. 2. TPE spectra of trans-stilbene: (a) two-photon polarization parameter $\Omega$; (b) excitation spectrum for linearly polarized photons of equal energy $\left(\delta_{\uparrow \uparrow} \eta\right.$ is in arbitrary units; $\eta$ is the fluorescence quantum yield).

pronounced in trans-indanylidenindane as a result of the more rigid skeleton) in accordance with the theoretical prediction that torsion of the phenyl rings up to about $30^{\circ}$ has only a minor influence on the calculated spectrum. The three bands in the UV spectrum of trans-stilbene are labelled I, II and III instead of the usual labelling $A, B$ and $C$ since the latter may cause confusion with the symmetry assignment.

The absorption polarization spectra of trans-stilbene and trans-indanylidenindane could be measured down to the beginning of band II (Fig. 3 ). Over the whole range of band I the degree $P$ of polarization is nearly constant and close to the limiting value of 0.5 for both compounds. This indicates that vibronic coupling via non-totally-symmetric vibrations only makes a small contribution to the intensity of band I. At the beginning of band II $P$ drops to a value of 0.1 . This value corresponds to an angle $\alpha$ between the transition moments of transitions I and II of about $45^{\circ} . \alpha$ is most probably even greater, since perturbations always reduce the absolute values of $P$. A similar result $\left(\alpha=53^{\circ}\right)$ was found by Yogev and Margulies [38] from measurements of the dichroism of $4,4^{\prime}$-dimethylstilbene embedded in stretched polyethylene foils. The decrease in $P$ at the beginning of band II is even more pronounced in trans-indanylidenindane. The measured $P$ value of -0.06 corresponds to an angle of about $60^{\circ}$. Band III again is polarized mainly in the direction of the long axis [38].

The TPE spectrum shows three distinct bands (labelled $a, b$ and c) below $44000 \mathrm{~cm}^{-1}$. Towards higher energies we observe some structure between 44000 and $47000 \mathrm{~cm}^{-1}$ (band d) and a strong increase in two-photon 
(b)

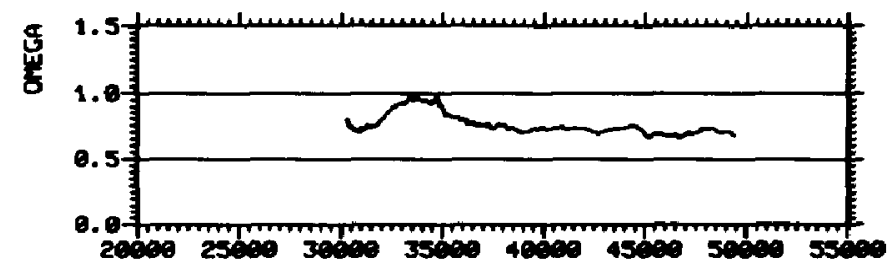

(a)

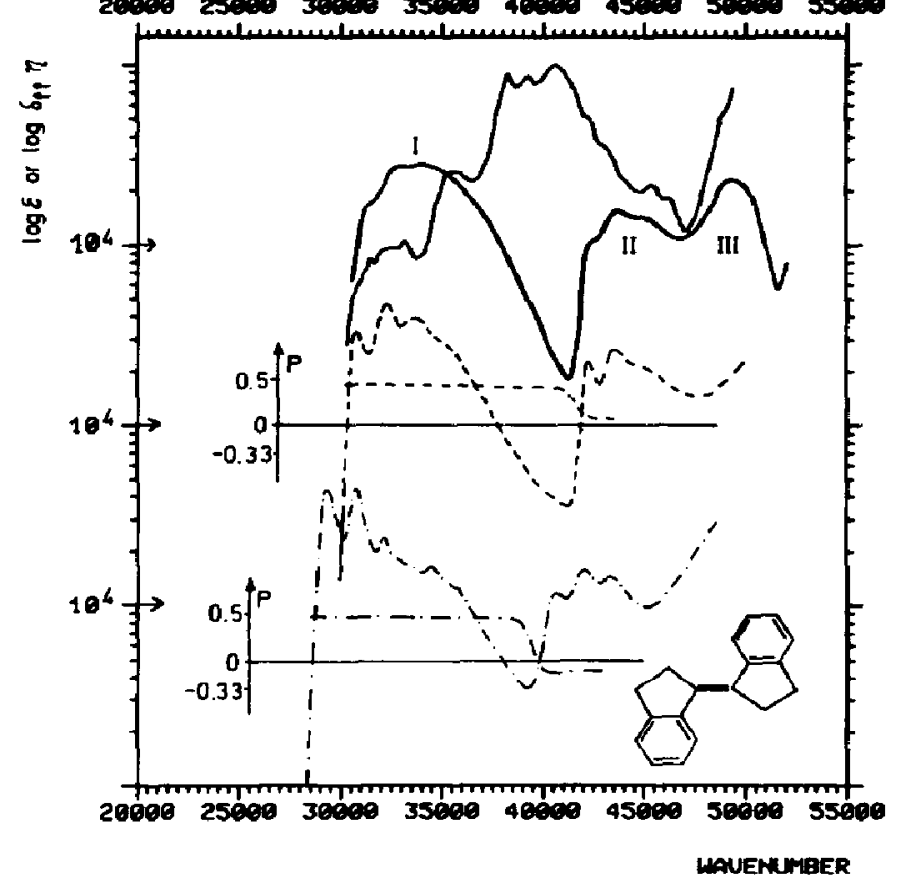

Fig. 3. (a) One- and two-photon absorption of trans-stilbene at room temperature (solvent, ethanol) (-) and absorption and absorption polarization spectra of trans-stilbene (- - -) and trans-indanylidenindane (--) at $77 \mathrm{~K}$ (solvent, 3-methylpentane); (b) twophoton polarization parameter (room temperature; solvent, ethanol).

absorption above $47000 \mathrm{~cm}^{-1}$ (e). The energy distance between this increase and the onset of band I corresponds perfectly to the onset of the transient absorption observed by Greene et al. [9].

The intense band $c$ around $40500 \mathrm{~cm}^{-1}$ has been observed in an early investigation by Stachelek et al. [19] and also later by Fuke et al. [20], but no structure was seen by these researchers. The absolute two-photon cross section at $39000 \mathrm{~cm}^{-1}$ was determined to be $12 \times 10^{-50} \mathrm{~cm}^{4} \mathrm{~s}$ (photon molecule) ${ }^{-1}$ [39]. This leads to about $14 \times 10^{-50} \mathrm{~cm}^{4} \mathrm{~s}$ (photon molecule) ${ }^{-1}$ for the maximum at $40500 \mathrm{~cm}^{-1}$, with the assumption of a constant fluorescence quantum yield $\eta$. However, Fuke et $a l$. [20] have compared the TPE spectrum with results obtained from the thermal lensing method. From this comparison, they concluded that the quantum yield drops by a factor of about 10 between 38000 and $44000 \mathrm{~cm}^{-1}$. From the data given by Fuke et $a l$. a band maximum at about $42000 \mathrm{~cm}^{-1}$ and a cross section of about $60 \times 10^{-50} \mathrm{~cm}^{4} \mathrm{~s}$ (photon molecule) ${ }^{-1}$ are estimated. 
Stachelek et al. [19] assigned the strong two-photon allowed band around $40500 \mathrm{~cm}^{-1}$ (band $\mathrm{c}$ in our notation) to the $1 \mathrm{~A}_{\mathrm{g}} \rightarrow 2 \mathrm{~A}_{\mathrm{g}}$ transition. This assignment was questioned by Orlandi et al. [17] since they find another low-lying $A_{\mathrm{g}}$ state in their theoretical study. Orlandi et al. [17] assign band $c$ to $1 \mathrm{~A}_{\mathrm{g}} \rightarrow 3 \mathrm{~A}_{\mathrm{g}}$. Our spectrum reveals two bands ( $\mathrm{a}$ and $\mathrm{b}$ ) at lower energies than band c. Most of the intensity of these two bands must result from transitions into A states since $\Omega$ remains below 1.0 over the whole spectrum. As discussed earlier, transitions to B states must lead to maxima in $\Omega$ (see Table 1 ). The shallow maximum in the $\Omega$ curve between 32500 and $35000 \mathrm{~cm}^{-1}$ may be due either to a $B$ state which gains some two-photon intensity because of the non-planarity of the molecule or to an A state for which the cross section is dominated by the $x y$ element of the two-photon transition tensor.

Finally we have to deal with the possibility that band a is not really connected with an excited state of symmetry $A$. Since the onsets of oneand two-photon absorption are very close, band a could in principle be related to $1 B$, the state responsible for band $I$ in the one-photon spectrum. If this is true, nearly all the two-photon intensity must result from vibronic coupling induced by $b$ vibrations. However, on the basis of the measured absolute $\delta$ value at $39000 \mathrm{~cm}^{-1}$ [39] the cross section found for band a exceeds $1 \times 10^{-50} \mathrm{~cm}^{4} \mathrm{~s}$ (photon molecule) ${ }^{-1}$. Such a value is fairly high for a purely vibronically induced two-photon transition. High resolution low temperature measurements are being carried out to clarify this subject further.

\section{Discussion}

To compare our experimental results with the outcome of theoretical investigations, we describe the low-lying excited states of stilbene in the framework of the exciton model [40]. A similar description has been used in the pioneering work of Dyck and McClure [13] and later also by Orlandi et al. [17]. In the exciton model the excited states of a molecule which consists of several well-defined subunits are described as linear combinations of the excited states of these subunits. To obtain sufficient quantitative results excitations from one subunit to the others must be included (charge exchange type of excitations) $[40,41]$ in addition to the local excitations. To characterize a low-lying excited state, however, it is usually sufficient to specify only the most important contributions from the local excitations. The main advantage of such a characterization is its independence of the calculated energetic order, a fact specifically helpful in comparing results of different theoretical treatments.

To establish how to characterize the low-lying excited states of transstilbene, we start with two benzene molecules in the correct orientation (Fig. 4(b)). If only interactions via long-range Coulomb forces are included, the resulting combinations of the local excited states $L_{b}, L_{a}$ and $B_{a, b}$ of benzene (Fig. 4(a)) split as shown in Fig. 4(b). The same nomenclature is 


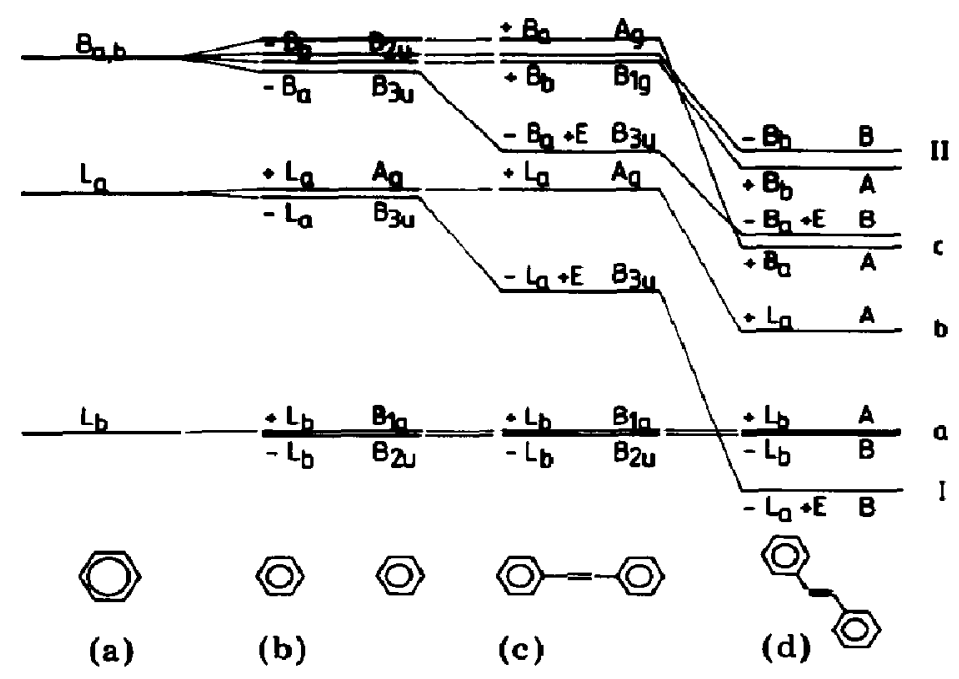

Fig. 4. Electronic energy levels of trans-stilbene as derived from those of two benzene molecules and ethylene. The symbols on the right-hand side show the final assignment.

used as in the paper of Orlandi et al. [17], e.g. $+L_{a}=2^{-1 / 2}\left\{L_{a}(p h e n y l ~ 1)+\right.$ $L_{a}$ (phenyl 2)\}. As long as $D_{2 h}$ symmetry is retained, the $\pi \pi^{*}$ excited states of the ethylene moiety $E$ can interact only with the $B_{3 u}$ combinations $-L_{a}$ and $-B_{a}$ (Fig. 4(c)). The states thus derived from the exciton model are then correlated with those obtained-from our SDCI/P $200 \pi \pi^{*}$ calculation (Fig. $4(d))$. Since the deviation from the idealized topological symmetry $D_{2 h}$ is not very large the mixing between the $B_{2 u}$ and $B_{3 u}$ states (which both become $B_{u}$ in $C_{2 h}$ symmetry) as well as that between the $B_{1 g}$ and $A_{g}$ states (which both become $A_{g}$ ) are not very strong. Correspondingly the calculated transition moments for the $B_{3 u}$-derived states are still oriented mainly parallel to the "long" axis of the molecule whereas the transition moments of $\mathbf{B}_{2 \mathrm{u}}$-derived states lie more parallel to the "short" axis. A similar result has been found in other calculations. The results of several calculations are listed in Table 4.

In all the calculations except those of Olbrich [18] the lowest excited $B$ state is $-L_{a}+E$. Since the calculated transition moments indicate $1 \mathrm{~A} \rightarrow$ $-L_{a}+E$ to be fully allowed this transition is usually assigned to band $I$. $-\mathrm{L}_{\mathrm{b}}$ can never be associated with this intense band since it is a combination of nearly unperturbed local $L_{b}$ states. The transition $1 A \rightarrow-L_{b}$ is expected to be weak and preferably polarized perpendicular to the long axis in contrast with the experimental findings [38].

In spite of the vigorous theoretical and experimental investigations of the one-photon spectrum of trans-stilbene the assignment of bands II and III still causes problems. In most of the more recent publications [14, 42 44] band II is assigned to $1 A \rightarrow-B_{a}+E$ and band III to $1 A \rightarrow-B_{b}$. Following this assignment band II should be polarized mainly parallel and band III more or less perpendicular to the long axis. This clearly contradicts the experimental observations discussed in Section 4 . We therefore have to look for a different explanation. 


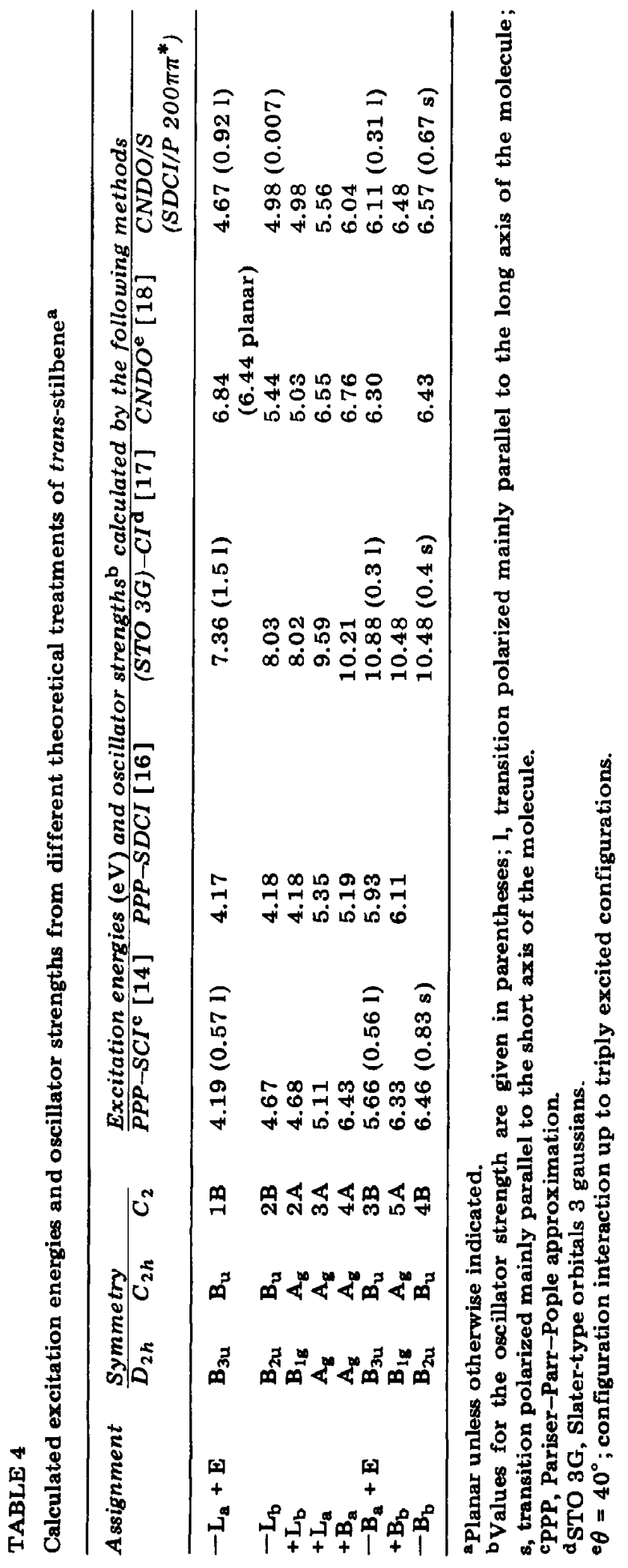


The only reasonable assignment which is in accordance with polarization in the direction of the short axis for band II is $1 A \rightarrow-B_{b}$. The calculated angle between the transition moments of $1 \mathrm{~A} \rightarrow-\mathrm{B}_{\mathrm{b}}$ and $1 \mathrm{~A} \rightarrow$ $-\mathrm{L}_{\mathrm{a}}+\mathrm{E}$ is $55^{\circ}$ for planar trans-stilbene. This value compares quite well with the experimental data.

For band III we are then left with two different possibilities.

(a) Band III results from $1 \mathrm{~A} \rightarrow-\mathrm{B}_{\mathrm{a}}+\mathrm{E}$. Such an assignment has been discussed in earlier investigations $[28,45]$ but among the more recent calculations only the $a b$ initio calculation of Orlandi et al. [17] with its unrealistic high excitation energies yields such a result.

(b) Band III is due to transitions to the states $5 \mathrm{~B}, 6 \mathrm{~B}$ and $7 \mathrm{~B}$. These evolve from the $1 \mathrm{~A}_{\mathrm{g}} \rightarrow 5 \mathrm{~B}_{\mathrm{u}}$ transition of the planar system (see Tables 2 and 3 ). All three are polarized mainly in the direction of the long axis.

As a consequence of assignment (b) it has to be assumed that band $I$ is a superposition of the transitions $1 \mathrm{~A} \rightarrow-\mathrm{L}_{\mathrm{a}}+\mathrm{E}$ and $1 \mathrm{~A} \rightarrow-\mathrm{B}_{\mathrm{a}}+\mathrm{E}$. Since both transitions are polarized parallel to the long axis such a superposition does not contradict the nearly constant polarization found for band $I$.

For the following reasons we favour assignment (b).

(i) If assignment (a) is correct, different semiempirical methods, which usually describe the order of low-lying one-photon allowed transitions of conjugated $\pi$ systems quite well, all predict the wrong sequence.

(ii) Assignment (b) is in accordance with the close energetic neighbourhood of the states $4 \mathrm{~A}\left(+\mathrm{B}_{\mathrm{a}}\right)$ and $3 \mathrm{~B}\left(-\mathrm{B}_{\mathrm{a}}+\mathrm{E}\right)$ predicted by our calculations.

(iii) Since $-B_{a}$ is the second combination of benzene states which strongly interacts with the $\pi \pi^{*}$ excited state of the ethylene moiety $1 \mathrm{~A} \rightarrow$ $-B_{a}+E$ (the second conjugation band in Suzuki's terminology [28]) is expected to lie at lower energies than $1 A \rightarrow-B_{b}$ and not at higher energies (Fig. 4).

From the low-lying excited states of symmetry B we are now only left with $-L_{b}$. There is no reason to assume that this state lies at higher energies than $L_{b}$ in benzene itself. This gives an upper limit of about $38000 \mathrm{~cm}^{-1}$ or $4.7 \mathrm{eV}$. Correspondingly most of the calculations yield $-L_{b}$ as the second $B$ state but the energetic distance from $1 B$ varies between nearly 0 and 0.5 $\mathrm{eV}$, when the unrealistic high energy values found by Orlandi et al. [17] are disregarded. Several hints can be found in the literature [15, 38], which confirm the existence of a weak transition polarized in the direction of the short axis, which is hidden under the strong band $I$.

For the states of symmetry $A$ the most convincing assignment is to correlate the intense two-photon absorption (band c) around $40500 \mathrm{~cm}^{-1}$ with the third excited $A$ state, $+B_{a}$. This differs from the assignment given by Orlandi et al. [17] who correlated this band with the second excited A state $+L_{a}$. However, only the experimental spectrum of Stachelek et al. [19] was available to Orlandi et al. [17] and they did not know that two other bands appear in the TPE spectrum below that around $40500 \mathrm{~cm}^{-1}$. In addition Orlandi et al. did not calculate two-photon cross sections. The 
qualitative estimate of two-photon intensities given by these researchers holds as well for $+L_{a}$ as it does for $+B_{a}$. In the Pariser-Parr-Pople approximation, however, $+L_{a}$ is a "plus" state. Thus the $1 A \rightarrow+L_{a}$ transition should be forbidden in the limit of perfect pairing $[36,46] .+B_{a}$, in contrast, is a "minus" state and the two-photon transition $1 \mathrm{~A} \rightarrow+\mathrm{B}_{\mathrm{a}}$ is allowed under pseudoparity selection rules [47].

If band $c$ is assigned to $1 \mathrm{~A} \rightarrow+\mathrm{B}_{\mathrm{a}}$ the natural assignment for band $\mathrm{b}$ is $1 \mathrm{~A} \rightarrow+\mathrm{L}_{\mathrm{a}}$. The ratio of the calculated two-photon cross sections as well as the calculated $\Omega$ values are in excellent agreement with experiment, especially if we take into account the observation of Fuke et al. [20].

Finally we have to deal with the assignment of band a. All calculations and even the old qualitative discussion of Dyck and McClure [13] predict the lowest excited A state to be $+L_{b}$, which again is a "minus" state. From our calculations we obtain a two-photon cross section of about $1 \times 10^{-50}$ $\mathrm{cm}^{4} \mathrm{~s}$ (photon molecule) $)^{-1}$ and an $\Omega$ value of 1.5 for the corresponding transition. The latter value clearly shows that the $x y$ element of the twophoton transition tensor is dominant in this case in accordance with the $B_{18}$ character of $+L_{b}$ in the topological $D_{2 h}$ symmetry. In the experiment we do not find an $\Omega$ value of 1.5 but we observe the shallow maximum between 32500 and $35000 \mathrm{~cm}^{-1}$, showing that there is at least some of the theoretically predicted intensity. We therefore conclude that most of the two-photon intensity observed between 32500 and $35000 \mathrm{~cm}^{-1}$ results from the transition $1 \mathrm{~A} \rightarrow+\mathrm{L}_{\mathbf{b}}$ but that the long wavelength part of band $a$ is due to vibronic coupling.

Because of the high density of calculated states with energies higher than $6 \mathrm{eV}$, we do not attempt to make definite assignments for structures $\mathrm{d}$ and e. Candidates are the higher excited $\mathrm{A}$ states $(6 \mathrm{~A}-10 \mathrm{~A})$ listed in Table 2. Because of the non-planarity of trans-stilbene in solution, strong mixing between $\sigma \pi^{*}$ and $\pi \pi^{*}$ excitations is probable in this energy range (the results for $\theta=0^{\circ}$ and $\theta=20^{\circ}$ should be compared in Tables 2 and 3 ). The closely spaced states $7 \mathrm{~A}-10 \mathrm{~A}$ account quite well for the strong increase in two-photon absorption observed above $47000 \mathrm{~cm}^{-1}(\mathrm{e})$. The calculated oscillator strength $\left(\theta=20^{\circ}\right)$ for a transition from the lowest excited singlet state $1 B$ into this group of $A$ states is about 0.1 . For all lower-lying $S_{1} \rightarrow S_{n}$ transitions except $S_{n} \equiv 4 \mathrm{~A}$ the calculated $f$ values are very small. The $f$ value calculated for $1 \mathrm{~B} \rightarrow 4 \mathrm{~A}$ is about 0.3 . Thus, a transient absorption should be detectable around $10000 \mathrm{~cm}^{-1}$ in addition to that observed around 20000 $\mathrm{cm}^{-1}$ [9].

\section{Implications to photochemistry}

The analysis given above requires a modification of some details of the OS model. As mentioned in Section 1 it is generally accepted that the lowest electronically excited state in the vicinity of the perpendicular conformation $\left(\phi=90^{\circ}\right)$ is an A state. As mentioned earlier this state is termed ${ }^{1} p^{* *}$ by Saltiel et al. [3]. In going from the perpendicular conformation 


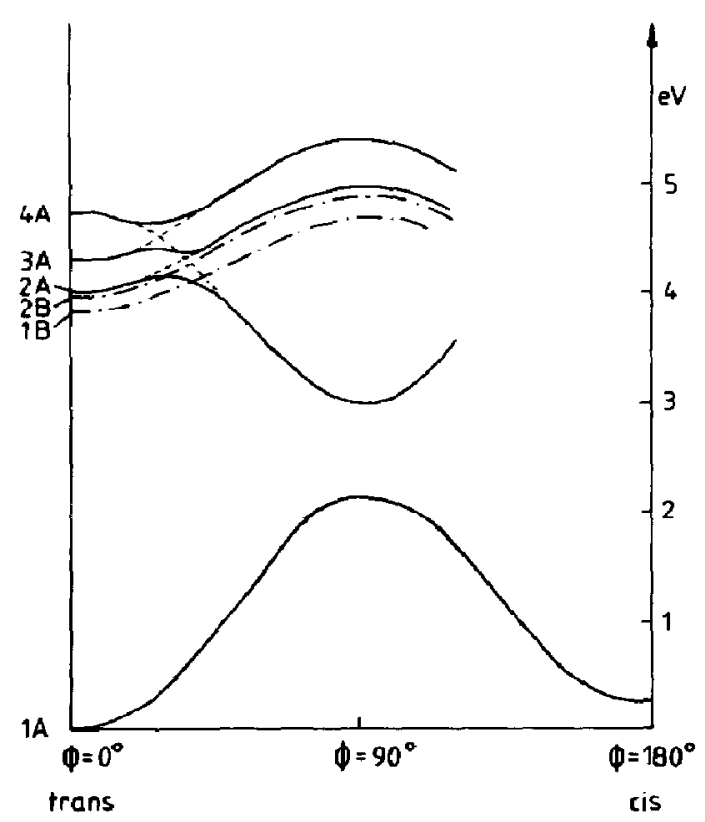

Fig. 5. Modified version of the $O S$ model. The curves for the excited states are not extended towards $\phi=180^{\circ}$ since enough experimental information is not yet available for the $c$ is form.

to either the trans or the cis form the energy of ${ }^{1} p^{* *}$ increases. However, to which excited state of these forms does ${ }^{1} p^{* *}$ really correspond? From our findings together with the results of the theoretical investigations it is most probable that ${ }^{1} p^{* *}$ corresponds to the third excited A state (4A) of trans-stilbene, as shown in Fig. 5. The calculations of Tavan and Schulten [16], Orlandi et $a l$. [17] and Olbrich [18] all show that the lowest excited A state (2A) increases in energy with increasing $\phi$ starting from $\phi=0^{\circ}$. A similar behaviour is indicated in the calculations of Tavan and Schulten [16] and Olbrich [18] for 3A. In our calculations $4 \mathrm{~A}$ is the first excited state which contains larger contributions of doubly excited configurations, contributions which are expected to become dominant in ${ }^{1} p^{* *}$. The most convincing argument in favour of a direct correlation between $4 \mathrm{~A}$ of the trans form and ${ }^{1} \mathrm{p}^{* *}$ is the observation of Fuke et al. [20] that direct population of $4 \mathrm{~A}$ leads to a drastic decrease in the fluorescence quantum yield.

The correlation of $4 A$ of the trans form with ${ }^{1} \mathrm{p}^{* *}$ leads to two avoided crossings between $\phi=0^{\circ}$ and $\phi=90^{\circ}$ which most probably introduce a barrier towards rotation around the central double bond in the lowest excited A state (2A) (Fig. 5). This immediately leads to the question raised in Section 1 about the nature of the experimentally observed barrier. If the 0-0 transitions of $1 \mathrm{~B}$ and $2 \mathrm{~A}$ are close together, as indicated by one calculation, the observed barrier may be directly related to the barrier in the lowest excited A state. If, however, $2 A$ lies about $2000 \mathrm{~cm}^{-1}$ above $1 \mathrm{~B}$, as estimated from our low resolution spectra, the barrier must result from the non-adiabatic crossing of $1 B$ and $2 A$. In this case the crossing must occur 
at relatively high twist angles $\phi$. To decide between these two possibilities it is absolutely necessary to localize precisely the $2 \mathrm{~A}$ state of the trans form.

\section{Acknowledgments}

The authors wish to express their gratitude towards the Deutsche Forschungsgemeinschaft and towards the Fonds der Chemischen Industrie for financial support. We also wish to thank the Rechenzentrum der Universität $\mathrm{zu}$ Köln for providing the necessary computer time and Mrs. B. Börsch-Pulm and Mr. P. F. Schönzart for their help with the calculations.

\section{References}

1 G. Orlandi and W. Siebrand, Chem. Phys. Lett., 30 (1975) 352.

2 J. Saltiel and J. L. Carlton, in P. de Mayo (ed.), Rearrangements in Ground and Excited States, Vol. 3, Academic Press, New York, 1980, p. 25.

3 J. Saltiel, J. D'Agostino, E. D. Megarity, L. Metts, K. R. Neuberger, M. Wrighton and O. C. ZaFiriou, Org. Photochem., 3 (1973) 1.

4 D. J. S. Birch and J. B. Birks, Chem. Phys. Lett., 38 (1976) 432.

5 O. Teschke, E. P. Ippen and G. R. Holtom, Chem. Phys. Lett., 52 (1977) 233.

6 M. Sumitani, N. Nagashima, K. Yoshihara and S. Nagakura, Chem. Phys. Lett., 51 (1977) 183.

7 R. Bonneau, J. Photochem., 10 (1979) 439.

8 B. I. Greene, R. M. Hochstrasser and R. B. Weisman, Chem. Phys. Lett., 62 (1979) 427.

9 B. I. Greene, R. M. Hochstrasser and R. B. Weisman, Chem. Phys., 48 (1980) 289.

10 H. P. Good, U. P. Wild, E. H. Fischer, E. P. Resewitz and E. Lippert, Ber. Bunsenges. Phys. Chem., 86 (1982) 126.

11 J. A. Syage, W. R. Lambert, P. M. Felker, A. H. Zewail and R. M. Hochstrasser, Chem. Phys. Lett., 88 (1982) 266.

12 M. Sumitani, N. Nakashima and K. Yoshihara, Chem. Phys. Lett., 68 (1979) 255.

M. Sumitani and K. Yoshihara, Bull. Chem. Soc. Jpn., 55 (1982) 85.

13 R. H. Dyck and D. S. McClure, J. Chem. Phys., 36 (1962) 2326.

14 D. L. Beveridge and H. H. Jaffe, J. Am. Chem. Soc., 87 (1965) 5340.

15 J. Bernstein, Spectrochim. Acta, Part A, 29 (1973) 147.

16 P. Tavan and K. Schulten, Chem. Phys. Lett., 56 (1978) 200.

17 G. Orlandi, P. Palmieri and G. Poggi, J. Am. Chem. Soc., 101 (1979) 3492.

18 G. Olbrich, Ber. Bunsenges. Phys. Chem., 86 (1982) 209.

19 T. M. Stachelek, T. A. Pazoha, W. M. McClain and R. P. Drucker, J. Chem. Phys., 66 (1977) 4540.

20 K. Fuke, S. A. Sakamoto, M. Ueda and M. Itoh, Chem. Phys, Lett., 74 (1980) 546.

21 F. Dörr, Angew. Chem., 78 (1966) 457.

22 W. Frölich, Dissertation, University of Cologne, Cologne, 1979.

23 J. Dehler and F. Dörr, Z. Angew. Phys., 19 (1965) 147.

24 B. Dick, H. Gonska and G. Hohlneicher, Ber. Bunsenges. Phys. Chem., 85 (1981) 746.

25 P. R. Monson and N. M. McClain, J. Chem. Phys., 53 (1970) 29; 56 (1972) 4817.

26 B. Dick and G. Hohlneicher, Chem. Phys. Lett., 83 (1981) 615;84 (1981) 471.

G. Hohlneicher and B. Dick, Pure Appl. Chem., 55 (1983) 261.

27 G. Hohlneicher and B. Dick, J. Chem. Phys., 70 (1979) 5427.

28 H. Suzuki, Bull. Chem. Soc. Jpn., 33 (1960) 379. 
29 M. Tratteberg and E. B. Frantsen, J. Mol. Struct., 26 (1975) 57.

30 J. Del Bene and H. H. Jaffe, J. Chem. Phys., 48 (1968) 1807, $4050 ; 49$ (1968) 1221 ; 50 (1969) 1126.

31 B. Dick and G. Hohlneicher, Theor, Chim. Acta, 53 (1979) 221.

32 K. Nishimoto and N. Mataga, Z. Phys. Chem., 12 (1957) 335; 13 (1957) 140.

33 R. Pariser, J. Chem. Phys., 21 (1953) 568.

R. Pariser and R. G. Parr, J. Chem. Phys., 21 (1953) 767.

34 R. L. Ellis, G. Kuehnlenz and H. H. Jaffe, Theor. Chim. Acta, 26 (1972) 131.

35 C. J. Finder, M. G. Newton and N. L. Allinger, Acta Crystallogr., Sect. B, 30 (1974) 411.

36 B. Dick and G. Hohlneicher, Chem. Phys. Lett., 97 (1983) 324.

37 R. L. Ellis and H. H. Jaffe, J. Mol. Spectrosc., 50 (1974) 474.

38 A. Yogev and L. Margulies, Isr. J. Chem., 16 (1977) 258.

39 R. J. M. Anderson, G. R. Holtom and W. M. McClain, J. Chem. Phys., 66 (1977) 3832 .

40 H. C. Longuet-Higgins and J. N. Murrell, Proc. Phys. Soc., London, Sect. A, 68 (1955) 601.

41 K. H. Giovanelli, G. Hohlneicher and P. A. Straub, Ber. Bunsenges. Phys. Chem., 75 (1971) 858.

42 H. Suzuki, Electronic Absorption Spectra and Geometry of Organic Molecules, Academic Press, New York, 1967, p. 306.

43 G. Favini, S. Fasone and M. Raimondi, Gazz. Chim. Ital., 97 (1967) 1434.

44 G. Wettermark, L. Tegner and O. Martensson, Ark. Kemi, 30 (1969) 185.

45 H. H. Perkampus and J. V. Knop, Theor. Chim. Acta, 6 (1966) 45.

46 P. R. Callis, T. W. Scott and A. C. Albrecht, J. Chem. Phys., 78 (1983) 16.

47 L. Goodman and R. P. Rara, Acc. Chem. Res., 17 (1984) 250. 